\title{
A sudden increase in delayed graft function in living donor kidney transplantation and a changed intraoperative fluid regimen.
}

Nieuwenhuijs-Moeke G.J. ${ }^{1}$, Huijnk T.M. ${ }^{2}$, Pol R.A. ${ }^{2}$, El Moumni M. ${ }^{2}$, Struys M.M.R.F. ${ }^{1}$, Berger S.P. ${ }^{3}$

Department of Anesthesiology ${ }^{1}$, Surgery ${ }^{2}$, Nephrology ${ }^{3}$, University of Groningen, University Medical Center Groningen, The Netherlands. Corresponding author: g.j.nieuwenhuijs-moeke@umcg.nl

\section{Introduction}

DGF:

Need for dialysis 1st week after transplantation functional DGF (fDGF): failure of fall in serum creatinin of $10 \%$ on 3 consecutive days 1 st week posttransplantation
2014: $1.4 \%$ DGF
2015: $\quad 4.4 \%$ DGF
$8.4 \%$ fDGF
2016: $11.3 \%$ DGF
$8.9 \%$ fDGF
$26.4 \%$ fDGF

\section{FLUID:}

2014: 4-5 L ballanced cristalloids for every recipient

2015: introduction GDFT, goal SVV $<10 \%$ at reperfusion

2016: GDFT, SVV $<10 \%$ at reperfusion for every recipient

Increase in DGF/fDGF related to adjustment in fluid regimen?

\section{Results}

Univariable analysis

Recipients with fDGF :
Donors were younger: 52 (41-60) vs 56 (47-64) P 0,0466 and taller:180 (173-184) vs173 (167-181)
Less preemptivly transplanted (more patients dialysis dependant): $15 \%$ vs $57 \%$
Intraoperative:
- less fluid ml total:
less $\mathrm{ml} / \mathrm{kg} \mathrm{BW:}$
more frequent noradrenaline:
more frequent noradrenaline low dose:
more frequent artery sacrificed
Lower urinairy output $1 \mathrm{st}$ :
Lower urinairy output 2nd h:

\section{Methods}

214 LDKT performed in UMCG between Jan 2014- June 2016:

\section{Data:}

Hospital records: donor, recipient characteristics

Digital patient data monitoring system: Intraoperative data

\section{Groups:}

fDGF: $n=26$, also includes patients meeting DGF criteria noDGF: $n=188$

\section{Analysis:}

univariable analysis

analysis over time

multivariable logistic regression

Changes in time
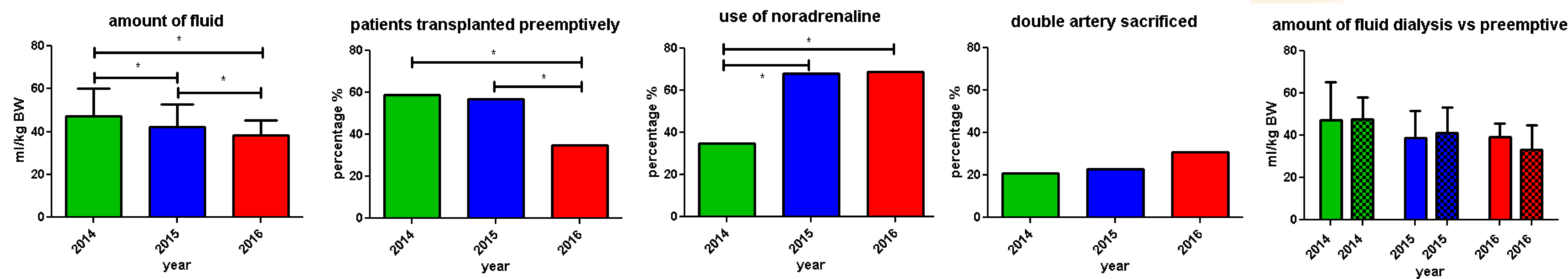

Logistic regression

Unadjusted analysis; Effect of the amount of fluid on developing fDGF was

$0.96(\mathrm{~B}-0,039,95 \% \mathrm{Cl}$ 0.932-0.993 P=0.016)

Adjusted for dialysis, sacrifice of an artery and the use of noradrenaline; Amount of fluid remained independently associated with fDGF

0,96 (B-0,037, 95\%Cl 0.931-0.997, $P=0.032$ )

\section{Conclusion}

Goal-directed fluid management towards an SVV of 10\% has led to reduced intraoperative fluid administration. This seems to be an independent risk factor for development of fDGF in living donor kidney transplantation. A more liberal fluid management using other goals might be more appropriate.

\section{Shared care for shared organs ${ }^{\circledR}$}

